\title{
Early Termination Clauses for Leasing Contracts with APR Cap
}

\author{
Luciano Quattrocchio ${ }^{1}$, Luisa Tibiletti ${ }^{1} \&$ Mariacristina Uberti ${ }^{1}$ \\ ${ }^{1}$ Department of Management, University of Torino, Torino, Italy \\ Correspondence: Mariacristina Uberti, Department of Management, University of Torino, Corso Unione \\ Sovietica 218/bis, 10134 Torino, Italy. E-mail: mariacristina.uberti@unito.it
}

Received: October 30, 2018

doi:10.5539/ijbm.v13n12p290
Accepted: November 23, 2018 Online Published: November 27, 2018

URL: https://doi.org/10.5539/ijbm.v13n12p290

\begin{abstract}
Terminating a leasing contract early may entail the payment of additional charges attributable to penalty and late payment costs. The occurrence of these extra charges push the lease effective Annual Percentage Rate (APR) up. The aim of this note is to discuss the contract early termination extra charge conditions which guarantee the no exceedance of a given APR threshold, whenever the contract expires. In the event the contract provides the lessee the option to terminate the lease prior to the first payment, extra charges shoot APR extraordinarily up and so the penalty costs should be set at zero. In the occurrence that the contract terminates upon the first payment date due to lessee's exercise of the early termination option, the most severe compliance conditions are those if the termination occurs at the first payment date. If the early termination occurs for lessee's insolvency, the most severe compliance condition is at correspondence of the first admissible date of the contract redemption for insolvency after a minimum number of unpaid payments. The late payment cost compliance condition requires only that the late payment interest rate not exceeds the given APR cap. Our findings hold whatever the payback amortization is set at the date of entering into the contract. If the French amortization (fixed installments) is used, the compliance constrains turn out looser than those valid for a general amortization.
\end{abstract}

Keywords: Annual Percentage Rate (APR), penalty costs, late payment fees.

\section{Introduction}

Many different reasons may lead a leasing contract to be expired prior the planned maturity. Early termination may occur if the lessee exercises the option of voluntary early termination or it comes out as a consequence of lessee's insolvency. Terminating the contract early implies the payment of extra costs. Usually, the lease contract terms require the payment of penalty costs calculated as a percentage of the outstanding debt and of late payment costs as extra interest on the no paid payments. The occurrence of extra charges pushes the effective Annual Percentage Rate (APR) up.

The aim of this note is to discuss the contract early termination extra charge conditions which guarantee that APR does not exceed a given threshold, whenever the contract will expire.

We show that if the lessee exercises the option to get out of the contract before the first payment date, the penalty costs may shoot APR extraordinarily up. So, in the event the contract provides the option to cease the lease prior to the first payment date, the penalty costs should be set at zero. If the contract provides the lessee to voluntary exercises the option of early termination only upon the first payment date, then it is just in correspondence of the first payment date that we find the most stringent early termination conditions. If early termination follows as a consequence of lessee's insolvency since the first payment date or insolvency after a previous period of regular payments, the most severe compliance condition is that at correspondence of the first admissible resolution date for insolvency. The only condition for the late payment costs is that the late payment interest rate is smaller than the given APR cap.

The relevance of our findings relies on the fact that the compliance formulae binding upon the penalty percentage on the outstanding debt at the resolution date hold for whatever the reimbursement plan is set at the date of entering into the contract. If the French amortization (fixed installments) is used, the compliance conditions are less stringent than those for general amortizations.

The remainder of the paper is organized as follows. In Section 2, the model is set up. In Section 3 the case of voluntary early resolution is considered. In Section 4 the case of early resolution caused by lessee's insolvency is analysed. Section 5 concerns repayment of lending with French amortization. Conclusions in Section 6 conclude 
the note. An Appendix collects the proofs.

\section{Notation and Layout of the Model}

We lay out the notation used throughout the paper. Let

- $\quad A$ be the leased amount, that is to be intended as the sum of the good price plus the initial costs needed to settle the contract;

- $\quad i$ be the annual compound lease contractual rate;

- $\quad n$ be the number of installments of the contract;

- $\quad R_{s} \geq 0$ be the installment due at the date $s$, with $s=1, \ldots, n$ to payback the initial debt $A$. Each $R_{s}$ is given by the sum of the capital share and the interest share. If the contract provides for a surrender value of the leased good to be paid at the date $n$, we assume that this amount is included in $R_{n}$;

- $z$ be the resolution date with $0<z \leq n$;

- $\quad k$ be the minimum number of unpaid installments after which the contract may be terminated for insolvency (Note 1);

- $D_{z}=\sum_{s=z+1}^{n} R_{s} \cdot(1+i)^{z-s}$ be the outstanding $\operatorname{debt}($ Note 2) at the resolution date $z$, after the payment of the installment $R_{s}$;

- $S(z)$ be the outflow at $z$;

- $m$ be the late payment interest rate. Usually the annual late payment interest rate is given as a nominal annual interest rate convertible the same number of times of the installments in a year. Without loss of generality, we indicate with $m$ the periodical compound interest rate with the same period of installments dates;

- $p$ be the penalty cost percentage on the outstanding debt;

- $\quad P_{z}=p \cdot D_{z}=p \cdot \sum_{s=z+1}^{n} R_{s} \cdot(1+i)^{z-s}$ be the penalty cost (Note 3) due to the voluntary resolution at the date $z$;

- $u$ be the effective APR cap, where $i<u$.

The contract may provide for a number of possible null installments. In general, we assume that

$$
R_{s} \geq 0 \text { for any } s=1, . ., n .
$$

As a consequence, some installments $R_{s}$ may not cover the correspondent interest share $i \cdot D_{s-1}$. Therefore, even in the case of regularly paid installments, it is not guaranteed that the outstanding debt $D_{s}$ is a decreasing function over time, but $D_{s}$ may be increasing over a period and decreasing after. The installments $R_{s}$, $s=1, \ldots, n$ solve the amortization initial closure condition, i.e.

$$
A=\sum_{s=1}^{n} R_{s} \cdot(1+i)^{-s} .
$$

The basic contractual conditions are assumed to hold, see for example Brealey et al. (2014), McConnell and Schallheim (1983) for the basic setting of an amortization plan, Yoshida et al. (2016, and the literature cited therein) for an interesting analysis of leasing contracts as well as of cancellable lease contracts that involve significant leasing costs.

\section{Voluntary Early Resolution}

Voluntary early resolution occurs when the lessee wishes to terminate his lease contract early before the end of the contracted term. Let $z$ be the resolution date that the lessee wants the contract out.

Assume that at the date $z$ the lessee is complaint with payments, therefore he has to effort only the penalties for the early contract termination but no additional late payment cost.

Penalties are given by the $p$ percent of the outstanding debt $D_{z}$ at the resolution date $z$. First, we consider the case that the resolution date be just after the contract inception and before the payment date of the first installment, i.e $z<1$. Second, we assume that the resolution date $z$ may be from the first date on, i.e. $1 \leq z<n$.

\subsection{Voluntary Resolution Before the First Payment Date}


Let assume that the date $z$ be just after the contract inception and before the first payment date, so $z<1$.

The outstanding debt at $z$, with $z<1$, is $D_{z}=A \cdot(1+i)^{z}$. In order that APR is capped by $u$, it is sufficient that the Net Present Value (NPV) at $u$ be positive:

$$
N P V(u)=A-(1+p) \cdot \frac{A \cdot(1+i)^{z}}{(1+u)^{z}}>0 \quad \text { with } z<1
$$

So

$$
p<\left(\frac{1+u}{1+i}\right)^{z}-1 \quad \text { with } z<1
$$

Since $i<u$ the right-hand side of (1) is positive and approaches zero as the resolution date $z$ tends to 0 . Then the sharpest threshold for $p$ is zero.

Result 1. Let the early resolution of the contract due to lessee's voluntary decision occur at the date $z$ with $z<1$ before the first payment date. Then APR is capped by the cap rate u for any resolution date $z$, with $z<1$, if

$$
p=0 \text {. }
$$

Result 1 shows that the contract would stipulate a minimum contract duration or abolish the penalty cost if the contract expires before the first payment date.

\subsection{Voluntary Resolution Upon the First Payment Date}

Let now suppose that the lessee wants the contract out at the date $z$, with $1 \leq z<n$.

If the desired resolution date is at correspondence of a payment date, i.e. $z$ is an integer number $z=1, . ., n-1$, the lessee is asked to pay the installment $R_{z}$ and the outstanding debt $D_{z}$ augmented by the penalty (Note 4) percent $p$.

The cash movements are given by the leased amount $A$ at the date 0 and the cash outflow $R_{1}, R_{2}, \ldots, R_{z-1}, S(z)$ at the dates $s=1, . ., z$ respectively, where

$$
S(z)=R_{z}+(1+p) \cdot D_{z}=R_{z}+(1+p) \cdot \sum_{s=z+1}^{n} \frac{R_{s}}{(1+i)^{s-z}} .
$$

In order that APR is capped by $u$ for any $z=1, . ., n-1$, it must be

$$
N P V(u)=A-\sum_{s=1}^{z-1} \frac{R_{s}}{(1+u)^{s}}-\frac{S(z)}{(1+u)^{z}}=A-\sum_{s=1}^{z} \frac{R_{s}}{(1+u)^{s}}-\frac{(1+p)}{(1+u)^{z}} \cdot \sum_{s=z+1}^{n} \frac{R_{s}}{(1+i)^{s-z}}>0
$$

Then for any $z=1, . ., n-1$

$$
p<\frac{A-\left[\sum_{s=1}^{z} \frac{R_{s}}{(1+u)^{s}}+\frac{1}{(1+u)^{z}} \cdot \sum_{s=z+1}^{n} \frac{R_{s}}{(1+i)^{s-z}}\right]}{\frac{1}{(1+u)^{z}} \cdot \sum_{s=z+1}^{n} \frac{R_{s}}{(1+i)^{s-z}}} .
$$

Result 2. Let the early resolution of the contract be due to lessee's voluntary decision. Then APR is capped by the cap rate $u$ for any resolution date $z$, with $1 \leq z<n$, if

$$
p<\frac{u-i}{1+i}
$$

corresponding to the compliance condition if the early resolution occurs at $z=1$ and $R_{1}=0$.

See Appendix A for the proof.

Remark 1. Condition (3) matches with intuition. If $R_{1}=0$ and the outstanding debt $D_{1}=A \cdot(1+i)$ is even 
higher than the leased amount $A$, it is intuitive to expect that the sharpest upper-bound for the penalty cost percentage $p$ occurs just in this situation. According to the intuition, the upper-bound (3) becomes looser as the cap $u$ goes up.

Remark 2. It is worthwhile noting that conditions (2) and (3) hold for any reimbursement plan, regardless of the installment stream of $R_{s}, s=1, . ., n$. Any type of amortization plan complaint with (2) and (3) has the effective APR capped by the cap rate $u$ for any possible resolution date $z$, with $1 \leq z<n$. If additional regularity assumptions on the installments' stream are introduced, the above conditions may be relaxed (see for example the case of French amortization in Sec.5).

\section{Early Resolution for Lessee's Insolvency}

Let assume that early resolution is a consequence of lessee's insolvency. First, we assume that the lesser is insolvent since the first cashable date. Second, we assume that the early resolution comes out after a period of insolvency started after a previous period of regular payments.

\subsection{A Bipole Cash-Flow}

Let assume that the lesser is insolvent since the first cashable date. The contract can be early terminated for insolvency only if at least $k$ payments are unpaid, so (Note 5) the resolution date $z$ is such that $k+1 \leq z \leq n-1$. Using the terminology introduced by Bornholt (2017), the cash flow reduces to a bipole financial operation being made up of the initial inflow of the leased amount $A$ and the outflow $S(z)$ at the termination date $z$ (see also Kulakov \& Kastro, 2017).

The final lump-sum outflow $S(z)$ due at the resolution date $z$ is the sum of three addenda:

$$
S(z)=M_{z}+D_{z}+P_{z} \quad \text { with } k+1 \leq z \leq n-1
$$

If the date $z$ is an integer number $z=k+1, . ., n-1$, the addenda can be written

- $M_{z}=\sum_{s=1}^{z} R_{s} \cdot(1+m)^{z-s}$ is the accumulated sum of the unpaid installments increased by the late payment cost and the installment $R_{z}$ due at $z$ without late payment cost;

- $D_{z}=\sum_{s=z+1}^{n} \frac{R_{s}}{(1+i)^{s-z}}$ is the outstanding debt at the date $z$, given by the present value at $z$ of the installments due from $z+1$ to $n$;

- $\quad P_{z}=p \cdot D_{z}=p \cdot \sum_{s=z+1}^{n} \frac{R_{s}}{(1+i)^{s-z}}$ is the penalty cost for early resolution.

As a bipole cash flow with final lump-sum $S(z)$ the contract effective APR is capped by the cap rate $u$, if and only if

$$
S(z)<A \cdot(1+u)^{z} \quad \text { for any } z=k+1, . ., n-1,
$$

where the both hand sides of (5) are increasing functions of $z$.

Re-writing (5)

$$
\sum_{s=1}^{z} R_{s} \cdot(1+m)^{z-s}+(1+p) \cdot \sum_{s=z+1}^{n} \frac{R_{s}}{(1+i)^{s-z}}<A \cdot(1+u)^{z} \quad \text { for any } z=k+1, . ., n-1
$$

then 


$$
p<\frac{A \cdot(1+u)^{z}-\left[\sum_{s=1}^{z} R_{s} \cdot(1+m)^{z-s}+\sum_{s=z+1}^{n} \frac{R_{s}}{(1+i)^{s-z}}\right]}{\sum_{s=z+1}^{n} \frac{R_{s}}{(1+i)^{s-z}}} \quad \text { for any } z=k+1, . ., n-1
$$

In the following we show that the sharpest threshold for $p$ is attained at $z=k+1$, i.e. when early resolution occurs after the first $k$ unpaid installments.

Result 3 Let the resolution for insolvency occur at the date $z$ as a consequence of at least $k$ unpaid installments. The effective APR is capped by the cap rate $u$ for any resolution date $z$, with $k+1 \leq z \leq n-1$ if the contract is compliant with the early resolution conditions for the first admissible resolution date $z=k+1$, specifically

- if $0 \leq m \leq i<u$ :

$$
\begin{gathered}
p<A \cdot \frac{(1+u)^{k+1}-(1+i)^{k+1}}{\sum_{s=k+2}^{n} \frac{R_{s}}{(1+i)^{s-(k+1)}}} \\
p<\left[A-\sum_{s=1}^{n} \frac{R_{s}}{(1+m)^{s}}\right] \cdot \frac{\left[(1+u)^{k+1}-(1+i)^{k+1}\right]}{\sum_{s=k+2}^{n} \frac{R_{s}}{(1+i)^{s-(k+1)}} .}
\end{gathered}
$$

See Appendix B.1 for the proof.

Remark 3. As for the case of the voluntary early resolution (see Remark 1), the upper bound (7.1) for $p$ corresponds to the case of a contract settled at the date 0 that provides for $R_{1}=R_{2}=\ldots=R_{k}=0$ that early terminates at $z=k+1$. So the most stringent compliance condition for the penalty cost percentage $p$ is at correspondence of an early terminated contract whose unpaid installments $R_{1}, R_{2}, \ldots, R_{k}$ are of amounts approaching zero(Note 6).

Remark 4. Conditions (7.1) and (7.2) hold for any reimbursement plan endowed with any installment stream of $R_{s}$ paid at the dates $s=1,2, . ., n$. Above conditions may be relaxed under additional regularity assumptions on the installments' stream, (see the case of French amortization in Sec.5).

If the resolution date $z$ is between two payment dates, the sharpest threshold for $p$ still remains that at $z=k+1$ (see Appendix B.1 for the proof).

\subsection{Insolvency Occurred after a Period of Regularly Payments}

Let assume that the lessee regularly pays the installments till a given epoch $r$, that is known since at the contract starting. Subsequently he becomes insolvent for at least $k$ installments, so the contract may be terminated at the date $z$ with $r+k+1 \leq z<n$. More precisely, let

- $\quad r$ be the number of the first $r$ regularly paid installments. Let assume that $r$ is known since the date 0 . If $r=0$ no regularly paid installments occur, so the case falls down with the previously discussed case of early termination due to insolvency discussed in Section 4.1;

- $\quad k$ be the minimum number of unpaid installments needed to terminate the contract for insolvency;

- $z$ be the resolution date due to insolvency with $r+k+1 \leq z \leq n-1$.

The final lump-sum outflow $S(z)$ due at the resolution date $z$ is the sum of three addenda:

$$
S(z)=M_{z}+D_{z}+P_{z} \quad \text { where } r+k+1 \leq z \leq n-1 .
$$

If the date $z$ is an integer number, the above addenda can be written

- $M_{z}=\sum_{s=r+1}^{z} R_{s} \cdot(1+m)^{z-s}$ is the sum of the unpaid installments from $r+1$ to $z-1$ increased by the late payment cost plus the due installment $R_{z}$ at $z$; 
- $D_{z}=\sum_{s=z+1}^{n} \frac{R_{s}}{(1+i)^{s-z}}$ is the outstanding debt at the date $z$, given by the present value at $z$ of the installments due from $z+1$ to $n$;

- $P_{z}=p \cdot D_{z}=p \cdot \sum_{s=z+1}^{n} \frac{R_{s}}{(1+i)^{s-z}}$ is the penalty cost for early resolution.

In order that APR is capped by $u$ for any $z$ with $r+k+1 \leq z \leq n-1$, it must be

$$
N P V(u)=A-\sum_{s=1}^{r} \frac{R_{s}}{(1+u)^{s}}-\frac{1}{(1+u)^{z}} \cdot \sum_{s=r+1}^{z} R_{s} \cdot(1+m)^{s-r}-\frac{1+p}{(1+u)^{z}} \cdot \sum_{s=z+1}^{n} \frac{R_{s}}{(1+i)^{s-z}}>0
$$

Then

$$
p<\frac{A-\left[\sum_{s=1}^{r} \frac{R_{s}}{(1+u)^{s}}+\frac{1}{(1+u)^{z}} \cdot \sum_{s=r+1}^{z} R_{s} \cdot(1+m)^{s-r}+\frac{1}{(1+u)^{z}} \cdot \sum_{s=z+1}^{n} \frac{R_{s}}{(1+i)^{s-z}}\right]}{\frac{1}{(1+u)^{z}} \cdot \sum_{s=z+1}^{n} \frac{R_{s}}{(1+i)^{s-z}}}
$$

for any $z=r+k+1, . ., n-1$.

If $r=0$ the lessee is insolvent since the first cashable date $z=1$, so the question falls within the early termination solvency case discussed in Sec. 4.1 and the most stringent condition for (10) is for $z=1$.

If $r=z$, there are no unpaid installments so the case reduces to the voluntary early termination discussed in Sec. 3.2. Let fix $r$ with $1 \leq r<z$, where $r$ is known at epoch 0 .

Result 4. Let $r$ with $1 \leq r<z$ be the number of regularly paid installments, where $r$ is known since the date 0 . The lessee becomes insolvent for at least $k$ installments and the contract breaks down at the date $z$, with $r+k+1 \leq z \leq n-1$, the effective APR is capped by the cap rate $u$ if the contract is compliant with the early resolution conditions at the date $z=r+k+1$, specifically

$$
\begin{gathered}
\text { - if } 0 \leq m \leq i<u: \quad \frac{(1+u)^{r+k+1}-(1+i)^{r+k+1}}{\sum_{s=r+k+2}^{n} \frac{R_{s}}{(1+i)^{s-(r+k+1)}}} \\
\text { - if } i<m<u: p<\left[A-\sum_{s=1}^{n} \frac{R_{s}}{(1+m)^{s}}\right] \cdot \frac{(1+u)^{r+k+1}-(1+i)^{r+k+1}}{\sum_{s=r+k+2}^{n} \frac{R_{s}}{(1+i)^{s-(r+k+1)}}}
\end{gathered}
$$

If the resolution date $z$ is between two payment dates, the sharpest threshold for $p$ still remains that at $z=r+k+1$ (see Appendix B.2 for the proof).

Remark 5. Conditions (11.1) and (11.2) for $z=r+k+1$, where the number $r$ of regularly paid installments is known at epoch 0 , are looser than those (7.1) and (7.2) for early resolution at $z=k+1$ for insolvency since the first installment (see Appendix B.2 for the proof).

Remark 6. Result 4 offers a general solution that holds for any reimbursement plan endowed with any installment stream of $R_{s}$ paid at the dates $s=1,2, . ., n$. Conditions (11.1) and (11.2) may become looser if regularity assumptions on the installments' stream are introduced (see for example the case of French amortization in Sec.5).

\section{French Amortization: Fixed Installments}

Let the leased amount $A$ be paid back according to the French amortization with fixed installments. Due to the 
regularity assumption of fixed installments of amount $R$, the constrains on $p$ may come out to be looser than those valid for any type of installments' stream given in expressed in Results 1, 2, 3, 4 .

Let the leased amount $A$ be paid back according to the French amortization with fixed installments $R$ at the yearly dates $s=1,2, . ., n$. The first installment is paid at the date 1 and $R_{1}=R_{2}, \ldots=R_{n}=R$ it follows

$$
R=\frac{A}{a_{\bar{n} \mid i}}
$$

where $a_{n \mid i}=\frac{1-(1+i)^{-n}}{i}$ is the present value of a unitary ordinary annuity.

Result 5. Let the payback of the leased amount A be placed according to the French amortization.

- Let the voluntary early resolution occur at the date $z$, with $z<1$. The effective APR is capped by the cap rate $u$ for any resolution date $z$, with $z<1$, if

$$
p=0 \text {. }
$$

- Let the voluntary early resolution occur at the date $z$, with $1 \leq z \leq n-1$. The effective APR is capped by the cap rate $u$ for any resolution date $z$, if the contract is compliant with the early resolution conditions at the date $z=1$ i.e.

$$
p<\frac{a_{n \mid i}}{a_{n-1 \mid i}} \cdot(u-i)
$$

where the condition (12) is looser than condition (3) that holds for any installment profile.

- Let the early resolution occur at the date $z$, with $k+1 \leq z \leq n-1$, for lessee's insolvency after at least $k$ unpaid payments. The effective APR is capped by the cap rate $u$ for any resolution date $z$, if the contract is compliant with the early resolution conditions at the first admissible resolution date for insolvency $z=k+1$ i.e.

$$
\begin{array}{ll}
\text { if } 0 \leq m \leq i<u: & p<\frac{a_{n \mid i}}{a_{n-(k+1) i}} \cdot\left[(1+u)^{k+1}-(1+i)^{k+1}\right] \\
\text { if } i<m<u: & p<\frac{\left(a_{n \mid i}-a_{n \mid m}\right)}{a_{n-(k+1) i}} \cdot\left[(1+u)^{k+1}-(1+i)^{k+1}\right] .
\end{array}
$$

- Let the early resolution occur for lessee's insolvency after a period of $r$ regularly paid installments, with $r$ known at epoch 0, and a subsequent period of at least $k$ unpaid payments. The effective APR is capped by the cap rate $u$ for any resolution date $z$, with $1 \leq z \leq n-1$, if the contract is compliant with the early resolution conditions at the first admissible resolution date for insolvency $z=r+k+1$ i.e.

$$
\begin{array}{ll}
\text { if } 0 \leq m \leq i<u: & p<\frac{a_{n \mid i}}{a_{n-(r+k+1) \mid i}} \cdot\left[(1+u)^{r+k+1}-(1+i)^{r+k+1}\right] \\
\text { if } i<m<u: & p<\frac{\left(a_{n \mid i}-a_{n \mid m}\right)}{a_{n-(r+k+1) \mid i}} \cdot\left[(1+u)^{r+k+1}-(1+i)^{r+k+1}\right] .
\end{array}
$$

See Appendix $\mathrm{C}$ for the proof.

\section{Conclusion}

The occurrence of extra charges incurred due to early lease termination pushes the lease effective APR up. The aim of this note is to discuss the contract early termination conditions which guarantee no exceedance of APR respect to a given threshold, whatever the termination date occurs. In the event the lessee has the option to terminate the lease prior to the first payment date we show that the penalty costs may shoot APR extraordinarily up, so the penalty should be set at zero. If the contract terminates upon the first payment date, as a consequence 
of lessee's exercise of the early termination option, the most severe compliance conditions are in correspondence of the first payment date. If the lessee becomes insolvent since the first payment date or after a period of regular payments, the most severe compliance condition is at the first early resolution date for insolvency. The late payment cost compliance conditions require that the late payment interest rate not exceeds the given APR cap. Our findings hold whatever the payback amortization is set at the date of entering into the contract. If the French amortization (fixed installments) is used, the compliance conditions are looser than those for a general amortization.

\section{Acknowledgments}

The Author is grateful to Prof. Bornholt for a fruitful discussion on bipole projects. Usual caveat applies.

\section{References}

Bornholt, G. (2017). What is an Investment Project's Implied Rate of Return? Abacus, 53(4), 513-526. https://doi.org/10.1111/abac.12093

Brealey, R. A., Myers, S., \& Allen, F. (2014). The Principles of Corporate Finance (11th ed.). New York: McGraw-Hill Irwin.

Kulakov, N. Y., \& Kastro, A. N. B. (2017). New Applications of the IRR Method in the Evaluation of Investment Projects. Proceedings of the 2017 Industrial and Systems Engineering Conference. Pittsburgh, Pennsylvania, USA.

McConnell, J. J., \& Schallheim, J. S. (1983). Valuation of asset leasing contracts. Journal of Financial Economics, 12(2), 237-261. https://doi.org/10.1016/0304-405X(83)90037-5

Tagliavini, G., Carretta, A., \& Nicolini, G. (2009). Pricing the lease during the contract: An in-progress approach to lease evaluation. Managerial Finance, 35(10), 841. https://doi.org/10.1108/03074350910984719

Yoshida, J., Seko, M., \& Sumita, K. (2016). The Rent Term Premium for Cancellable Leases. The Journal of Real Estate Finance and Economics, 52(10), 480-511. https://doi.org/10.1007/s11146-015-9528-x

\section{Notes}

Note 1. The minimum number of unpaid installments after which the contract can be terminated depends on the jurisprudence. In Italy it is $k=6$ if the contract provides for monthly installments.

Note 2. In the current version we assume that the repayment cash flow incorporates the market value at any time. An admissible extension concerns the possibility of a rescue price, see Tagliavini et al. (2009).

Note 3. Note that no penalty cost overcharges the instalment $R_{z}$ owed at $z$.

Note 4. If $z=n$ no penalties occur because the termination date coincides with the lease maturity.

Note 5. If $z=n$ the contract expires with the lump-sum $S(n)=M_{n}$ and $P_{n}=0$ and $D_{n}=0$.

Note 6. If the contract expires at $z=k+1$ and $R_{1}=R_{2}=\ldots=R_{k}=0$, the cause of the early resolution is not imputable to lessee's insolvency.

\section{Appendix A}

\section{Voluntary resolution upon the first payment date}

Condition (2) for any integer date $z=1, . ., n-1$,

$$
p<\frac{A-\left[\sum_{s=1}^{z} \frac{R_{s}}{(1+u)^{s}}+\frac{1}{(1+u)^{z}} \cdot \sum_{s=z+1}^{n} \frac{R_{s}}{(1+i)^{s-z}}\right]}{\frac{1}{(1+u)^{z}} \cdot \sum_{s=z+1}^{n} \frac{R_{s}}{(1+i)^{s-z}}}
$$

Multiplying the numerator and the denominator by $(1+u)^{z}$, the upper-bound of (a.1) becomes 


$$
\frac{(1+u)^{z} \cdot\left[A-\sum_{s=1}^{z} \frac{R_{s}}{(1+u)^{s}}\right]-\sum_{s=z+1}^{n} \frac{R_{s}}{(1+i)^{s-z}}}{\sum_{s=z+1}^{n} \frac{R_{s}}{(1+i)^{s-z}}}
$$

We will prove that (a.2) is lower bound by an increasing function $f(z)$.

By definition the leased amount $A$ must be equal to the present value of the installments, i.e. $A=\sum_{s=1}^{z} \frac{R_{s}}{(1+i)^{s}}+\sum_{s=z+1}^{n} \frac{R_{s}}{(1+i)^{s}}$. Since $i<u, \frac{1}{1+i}>\frac{1}{1+u}$ then $\sum_{s=1}^{z} \frac{R_{s}}{(1+i)^{s}} \geq \sum_{s=1}^{z} \frac{R_{s}}{(1+u)^{s}}$. So the factor of the first addendum in the numerator of (a.2) is lower bound by

$$
\begin{aligned}
& {\left[A-\sum_{s=1}^{z} \frac{R_{s}}{(1+u)^{s}}\right]=\left(\sum_{s=1}^{z} \frac{R_{s}}{(1+i)^{s}}+\sum_{s=z+1}^{n} \frac{R_{s}}{(1+i)^{s}}\right)-\sum_{s=1}^{z} \frac{R_{s}}{(1+u)^{s}} \geq} \\
& \sum_{s=1}^{z} \frac{R_{s}}{(1+u)^{s}}+\sum_{s=z+1}^{n} \frac{R_{s}}{(1+i)^{s}}-\sum_{s=1}^{z} \frac{R_{s}}{(1+u)^{s}}=\sum_{s=z+1}^{n} \frac{R_{s}}{(1+i)^{s}}
\end{aligned}
$$

Then the numerator of (a.2) is lower bound by

$$
\begin{aligned}
& (1+u)^{z} \cdot\left[A-\sum_{s=1}^{z} \frac{R_{s}}{(1+u)^{s}}\right]-\sum_{s=z+1}^{n} \frac{R_{s}}{(1+i)^{s-z}} \geq \\
& (1+u)^{z} \cdot \sum_{s=z+1}^{n} \frac{R_{s}}{(1+i)^{s}}-(1+i)^{z} \cdot \sum_{s=z+1}^{n} \frac{R_{s}}{(1+i)^{s}}= \\
& =\left[(1+u)^{z}-(1+i)^{z}\right] \cdot \sum_{s=z+1}^{n} \frac{R_{s}}{(1+i)^{s}}
\end{aligned}
$$

Therefore (a.2) is lower bound as it follows

$$
\begin{aligned}
& \frac{(1+u)^{z} \cdot\left[A-\sum_{s=1}^{z} \frac{R_{s}}{(1+u)^{s}}\right]-(1+i)^{z} \cdot \sum_{s=z+1}^{n} \frac{R_{s}}{(1+i)^{s}}}{\sum_{s=z+1}^{n} \frac{R_{s}}{(1+i)^{s-z}}} \geq \\
& \frac{\left[(1+u)^{z}-(1+i)^{z}\right] \cdot \sum_{s=z+1}^{n} \frac{R_{s}}{(1+i)^{s}}}{(1+i)^{z} \cdot \sum_{s=z+1}^{n} \frac{R_{s}}{(1+i)^{s}}}=\frac{\left[(1+u)^{z}-(1+i)^{z}\right]}{(1+i)^{z}}=\left(\frac{1+u}{1+i}\right)^{z}-1
\end{aligned}
$$

Since $\frac{1+u}{1+i}>1$, the lower bound $f(z)=\left(\frac{1+u}{1+i}\right)^{z}-1$ is increasing in $z$, so its minimum is achieved at $z=1$.

It means that the most severe condition for the penalty cost percentage $p$ is lower bound by $f(1)=\left(\frac{1+u}{1+i}\right)-1=\frac{u-i}{1+i}$. It follows that

$$
\frac{u-i}{1+i} \leq \frac{(1+u)^{z} \cdot\left[A-\sum_{s=1}^{z} \frac{R_{s}}{(1+u)^{s}}\right]-(1+i)^{z} \cdot \sum_{s=z+1}^{n} \frac{R_{s}}{(1+i)^{s}}}{\sum_{s=z+1}^{n} \frac{R_{s}}{(1+i)^{s-z}}} \text { for every } z=1, . ., n-1
$$


To measure the difference between the right-hand side of (a.3) and its lower proxy $f(1)=\frac{u-i}{1+i}$ we substitute $z=1$ in the right-hand side of (a.3) and we get

$$
\frac{(1+u) \cdot\left[A-\frac{R_{1}}{1+u}\right]-(1+i) \cdot \sum_{s=2}^{n} \frac{R_{s}}{(1+i)^{s}}}{\sum_{s=2}^{n} \frac{R_{s}}{(1+i)^{s-1}}}=\frac{A \cdot(1+u)-\left[R_{1}+\sum_{s=2}^{n} \frac{R_{s}}{(1+i)^{s-1}}\right]}{\sum_{s=2}^{n} \frac{R_{s}}{(1+i)^{s-1}}}
$$

Since $\left[R_{1}+\sum_{s=2}^{n} \frac{R_{s}}{(1+i)^{s-1}}\right]=\sum_{s=1}^{n} \frac{R_{s}}{(1+i)^{s-1}}=(1+i) \cdot \sum_{s=1}^{n} \frac{R_{s}}{(1+i)^{s}}=(1+i) \cdot A$, we substitute and obtain

$$
=\frac{A \cdot(1+u)-A \cdot(1+i)}{\sum_{s=2}^{n} \frac{R_{s}}{(1+i)^{s-1}}}=\frac{A \cdot(u-i)}{\sum_{s=2}^{n} \frac{R_{s}}{(1+i)^{s}} \cdot(1+i)}=\frac{A}{\sum_{s=2}^{n} \frac{R_{s}}{(1+i)^{s}}} \cdot \frac{u-i}{1+i} .
$$

Note that the above coincides with $\frac{u-i}{1+i}$ if $\frac{A}{\sum_{s=2}^{n} \frac{R_{s}}{(1+i)^{s}}}=1$, i.e. $R_{1}=0$.

Then inequality (a.3) reduces to an equality for $z=1$ and $R_{1}=0$.

So the most severe condition for the penalty cost percentage $p$

$$
p<\frac{u-i}{1+i}
$$

corresponds to the case that early resolution occurs at $z=1$ and $R_{1}=0$.

If the resolution date $z$ is between two payment dates, the sharpest upper-bound for $p$ still remains at $z=1$, because the low proxy $f(z)$ is increasing in $z$.

\section{Appendix B}

\section{Early resolution for insolvency}

\section{B.1 Bipole cash flow}

We will show that the most severe condition for the penalty cost percentage $p$ is in correspondence of the minimum of the low proxy $f(z)$ at the date $z=k+1$. First, we assume that the resolution date be an integer number $z=k+1, \ldots, n-1$, then we extend the result to any $z$, with $k+1 \leq z<n$.

Let consider the following cases:

1. Late payment cost: $0 \leq m<i<u$.

- If $m=0$, condition (6) becomes

$$
p<\frac{A \cdot(1+u)^{z}-\left[\sum_{s=1}^{z} R_{s}+\sum_{s=z+1}^{n} \frac{R_{s}}{(1+i)^{s-z}}\right]}{\sum_{s=z+1}^{n} \frac{R_{s}}{(1+i)^{s-z}}} \quad \text { for any } z=k+1, . ., n-1
$$

Since $\left[\sum_{s=1}^{z} R_{s}+\sum_{s=z+1}^{n} \frac{R_{s}}{(1+i)^{s-z}}\right] \leq \sum_{s=1}^{z} R_{s} \cdot(1+i)^{z-s}+\sum_{s=z+1}^{n} \frac{R_{s}}{(1+i)^{s-z}}=A \cdot(1+i)^{z}$ 
then $-\left[\sum_{s=1}^{z} R_{s}+\sum_{s=z+1}^{n} \frac{R_{s}}{(1+i)^{s-z}}\right] \geq-A \cdot(1+i)^{z}$

The right-hand side of (b.1) is lower bound by

$$
\frac{A \cdot(1+u)^{z}-\left[\sum_{s=1}^{z} R_{s}+\sum_{s=z+1}^{n} \frac{R_{s}}{(1+i)^{s-z}}\right]}{\sum_{s=z+1}^{n} \frac{R_{s}}{(1+i)^{s-z}}} \geq A \cdot \frac{(1+u)^{z}-(1+i)^{z}}{\sum_{s=z+1}^{n} \frac{R_{s}}{(1+i)^{s-z}}}
$$

Since $i<u$, the lower proxy function $g(z)=A \cdot \frac{(1+u)^{z}-(1+i)^{z}}{\sum_{s=z+1}^{n} \frac{R_{s}}{(1+i)^{s-z}}}$ is increasing in $z$ and has its minimum at $z=k+1$. A lower proxy of the most stringent condition for $p$ is

$$
g(k+1)=A \cdot \frac{(1+u)^{k+1}-(1+i)^{k+1}}{\sum_{s=k+2}^{n} \frac{R_{s}}{(1+i)^{s-(k+1)}}}
$$

So the most stringent condition is

$$
p<A \cdot \frac{(1+u)^{k+1}-(1+i)^{k+1}}{\sum_{s=k+2}^{n} \frac{R_{s}}{(1+i)^{s-(k+1)}}}
$$

- If $0<m<i<u$, we show that the upper-bound of (6) is still lower bound by $g(z)=A \cdot \frac{(1+u)^{z}-(1+i)^{z}}{\sum_{s=z+1}^{n} \frac{R_{s}}{(1+i)^{s-z}}}$

Since $\left[\sum_{s=z}^{n} R_{s} \cdot(1+m)^{z-s}+\sum_{s=z+1}^{n} \frac{R_{s}}{(1+i)^{s-z}}\right] \leq \sum_{s=1}^{z} R_{s} \cdot(1+i)^{z-s}+\sum_{s=z+1}^{n} \frac{R_{s}}{(1+i)^{s-z}}=A \cdot(1+i)^{z}$, then

$$
\frac{A \cdot(1+u)^{z}-\left[\sum_{s=z}^{n} R_{s} \cdot(1+m)^{z-s}+\sum_{s=z+1}^{n} \frac{R_{s}}{(1+i)^{s-z}}\right]}{\sum_{s=z+1}^{n} \frac{R_{s}}{(1+i)^{s-z}}} \geq A \cdot \frac{(1+u)^{z}-(1+i)^{z}}{\sum_{s=z+1}^{n} \frac{R_{s}}{(1+i)^{s-z}}}
$$

Analogously to the previous case, the most severe condition for the penalty cost percentage $p$ is at correspondence of $g(k+1)$

$$
p<A \cdot \frac{(1+u)^{k+1}-(1+i)^{k+1}}{\sum_{s=k+2}^{n} \frac{R_{s}}{(1+i)^{s-(k+1)}}}
$$

- $\quad$ Late payment cost: $m=i$.

As known $A \cdot(1+i)^{z}=\sum_{s=1}^{z} R_{s} \cdot(1+i)^{z-s}+\sum_{s=z+1}^{n} \frac{R_{s}}{(1+i)^{s-z}}$, then condition (6) becomes 


$$
p<\frac{A \cdot(1+u)^{z}-\left[\sum_{s=1}^{z} R_{s} \cdot(1+i)^{z-s}+\sum_{s=z+1}^{n} \frac{R_{s}}{(1+i)^{s-z}}\right]}{\sum_{s=z+1}^{n} \frac{R_{s}}{(1+i)^{s-z}}}=A \cdot \frac{(1+u)^{z}-(1+i)^{z}}{\sum_{s=z+1}^{n} \frac{R_{s}}{(1+i)^{s-z}}}
$$

Analogously to the previous cases, the most severe condition for the penalty cost percentage $p$ is at correspondence of $g(k+1)$, then

$$
p<A \cdot \frac{(1+u)^{k+1}-(1+i)^{k+1}}{\sum_{s=k+2}^{n} \frac{R_{s}}{(1+i)^{s-(k+1)}}}
$$

- In conclusion for $0 \leq m \leq i<u$ the most severe condition for the penalty cost percentage $p$ is at correspondence of $g(k+1)$, then

$$
p<A \cdot \frac{(1+u)^{k+1}-(1+i)^{k+1}}{\sum_{s=k+2}^{n} \frac{R_{s}}{(1+i)^{s-(k+1)}}}
$$

Above condition coincides with the minimum of the right-hand side of (b.1) in the case of $z=k+1$ and $R_{1}=R_{2}=\ldots=R_{k}=0$. So the most stringent compliance condition for the penalty cost percentage $p$ is at correspondence of an early terminated contract at $z=k+1$ characterized by insolvent installments $R_{1}, R_{2}, \ldots, R_{k}$ whose amounts are approaching zero.

- $\quad$ Late payment cost: $i<m<u$

As known $A \cdot(1+i)^{z}=\sum_{s=1}^{z} R_{s} \cdot(1+i)^{z-s}+\sum_{s=z+1}^{n} \frac{R_{s}}{(1+i)^{s-z}}$, then the upper-bound for $p$ in (6) can be re-written

$$
\begin{aligned}
& \frac{A \cdot(1+u)^{z}-\left[\sum_{s=1}^{z} R_{s} \cdot(1+m)^{z-s}+\sum_{s=z+1}^{n} \frac{R_{s}}{(1+i)^{s-z}}\right]}{\sum_{s=z+1}^{n} \frac{R_{s}}{(1+i)^{s-z}}}= \\
& =\frac{\left[A \cdot(1+u)^{z}-A \cdot(1+i)^{z}\right]+A \cdot(1+i)^{z}-\left[\sum_{s=1}^{z} R_{s} \cdot(1+m)^{z-s}+\sum_{s=z+1}^{n} \frac{R_{s}}{(1+i)^{s-z}}\right]}{\sum_{s=z+1}^{n} \frac{R_{s}}{(1+i)^{s-z}}}= \\
& =\frac{\left[A \cdot(1+u)^{z}-A \cdot(1+i)^{z}\right]+\left[\sum_{s=1}^{z} R_{s} \cdot(1+i)^{z-s}+\sum_{s=z+1}^{n} \frac{R_{s}}{(1+i)^{s-z}}\right]-\left[\sum_{s=1}^{z} R_{s} \cdot(1+m)^{z-s}+\sum_{s=z+1}^{n} \frac{R_{s}}{(1+i)^{s-z}}\right]}{\sum_{s=z+1}^{n} \frac{R_{s}}{(1+i)^{s-z}}}
\end{aligned}
$$

Simplifying by $\sum_{s=z+1}^{n} \frac{R_{s}}{(1+i)^{s-z}}$ in the numerator, above reduces 


$$
\begin{aligned}
& \frac{\left[A \cdot(1+u)^{z}-A \cdot(1+i)^{z}\right]+\sum_{s=1}^{z} R_{s} \cdot\left[(1+i)^{z-s}-(1+m)^{z-s}\right]}{\sum_{s=z+1}^{n} \frac{R_{s}}{(1+i)^{s-z}}}= \\
&= \frac{\left[A \cdot(1+u)^{z}-A \cdot(1+i)^{z}\right]+\sum_{s=1}^{z} R_{s} \cdot\left[\frac{(1+i)^{z}}{(1+i)^{s}}-\frac{(1+m)^{z}}{(1+m)^{s}}\right]}{\sum_{s=z+1}^{n} \frac{R_{s}}{(1+i)^{s-z}}} \geq \\
& \frac{\left[A \cdot(1+u)^{z}-A \cdot(1+i)^{z}\right]+\sum_{s=1}^{z} R_{s} \cdot\left[\frac{(1+i)^{z}}{(1+m)^{s}}-\frac{(1+u)^{z}}{(1+m)^{s}}\right]}{\sum_{s=z+1}^{n} \frac{R_{s}}{(1+i)^{s-z}}} \geq \\
& \frac{\left[A \cdot(1+u)^{z}-A \cdot(1+i)^{z}\right]+\sum_{s=1}^{z} R_{s} \cdot\left[\frac{(1+i)^{z}-(1+u)^{z}}{(1+m)^{s}}\right]}{\sum_{s=z+1}^{n} \frac{R_{s}}{(1+i)^{s-z}}}= \\
&= \frac{A \cdot\left[(1+u)^{z}-(1+i)^{z}\right]-\left[(1+u)^{z}-(1+i)^{z}\right] \cdot \sum_{s=1}^{z} \frac{R_{s}}{(1+m)^{s}}}{\sum_{s=z+1}^{n} \frac{R_{s}}{(1+i)^{s-z}}}= \\
&= \frac{\left[(1+u)^{z}-(1+i)^{z}\right]}{\sum_{s=z+1}^{n} \frac{R_{s}}{(1+i)^{s-z}} \cdot\left[A-\sum_{s=1}^{z} \frac{R_{s}}{(1+m)^{s}}\right] \geq} \\
& \frac{R_{s}}{(1+i)^{s-z}} \cdot\left[A-\sum_{s=1}^{n} \frac{R_{s}}{(1+m)^{s}}\right]=g(z) \cdot\left[A-\sum_{s=1}^{n} \frac{R_{s}}{(1+m)^{s}}\right]
\end{aligned}
$$

where $g(z)$ has already proved to be an increasing function in $z$ and $\left[A-\sum_{s=1}^{n} \frac{R_{s}}{(1+m)^{s}}\right]$ is a positive constant, therefore (b.4) is increasing in $z$ and reaches its minimum at correspondence of $z=k+1$, the sharpest upper-bound for $p$ in (b.1) is

$$
p<\left[A-\sum_{s=1}^{n} \frac{R_{s}}{(1+m)^{s}}\right] \cdot \frac{\left[(1+u)^{k+1}-(1+i)^{k+1}\right]}{\sum_{s=k+2}^{n} \frac{R_{s}}{(1+i)^{s-(k+1)}}} \text { for any } m, \text { with } m<u
$$

If the resolution date $z$ is between two payment dates, the sharpest upper-bound for $p$ is at $z=k+1$, because if $0 \leq m \leq i$ the low proxy $f(z)$ is increasing in $z$; if $i<m<u$ the upper-bound for $p$ is increasing in $z$.

\section{Appendix B.2}

\section{Insolvency after a period of regularly paid installments}

Let the first $r$ installments, with $0<r \leq n-1$ be regularly paid. Assume that early resolution occurs for 
insolvency at the date $z$, with $r+k+1 \leq z \leq n-1$ whatever $k$ unpaid installments occur.

Substituting $r+k$ to $k$ and following the same reasoning of the proof in Appendix B.1, we get

- if $0 \leq m \leq i<u$ :

$$
\begin{aligned}
& p<A \cdot \frac{(1+u)^{r+k+1}-(1+i)^{r+k+1}}{\sum_{s=r+k+2}^{n} \frac{R_{s}}{(1+i)^{s-(r+k+1)}}} \\
& p<\left[A-\sum_{s=1}^{n} \frac{R_{s}}{(1+m)^{s}}\right] \cdot \frac{(1+u)^{r+k+1}-(1+i)^{r+k+1}}{\sum_{s=r+k+2}^{n} \frac{R_{s}}{(1+i)^{s-(r+k+1)}}}
\end{aligned}
$$

- if $i<m<u$ :

Above conditions concerning early resolution at $z=r+k+1$ are looser than the early resolution at $z=k+1$ due to insolvency since the first cashable date, because the low proxy of the upper-bound of $p$ and the upper-bound of $p$ are both increasing in the date $z$.

\section{Appendix C}

\section{The French amortization}

- Let the early resolution occur at the date $z$, with $z<1$. According to Result 1 effective APR is capped by the cap rate $u$ for any resolution date $z$, with $z<1$, if

$$
p=0 .
$$

- Let early resolution occur at the date $z$, with $1 \leq z \leq n-1$.

The upper-bound of $p$ given by (a.2) becomes

$$
\begin{aligned}
& \frac{(1+u)^{z} \cdot\left[A-\sum_{s=1}^{z} \frac{R}{(1+u)^{s}}\right]-\sum_{s=z+1}^{n} \frac{R}{(1+i)^{s-z}}}{\sum_{s=z+1}^{n} \frac{R}{(1+i)^{s-z}}}=\frac{(1+u)^{z} \cdot\left[a_{n \mid i}-a_{z \mid u}\right]-a_{n-z \mid i}}{a_{n-z \mid i}}= \\
& =\frac{(1+u)^{z} \cdot\left[a_{n \mid i}-a_{z \mid u}\right]}{a_{n-z \mid i}}-1=\frac{(1+u)^{z} \cdot a_{n \mid i}-\frac{(1+u)^{z}-1}{u}}{a_{n-z \mid i}}-1= \\
& =\frac{(1+u)^{z} \cdot\left[a_{n \mid i}-\frac{1}{u}\right]+\frac{1}{u}}{a_{n-z \mid i}}-1
\end{aligned}
$$

Since $g(z)=\frac{(1+u)^{z} \cdot\left[a_{n \mid i}-\frac{1}{u}\right]+\frac{1}{u}}{a_{n-z \mid i}}-1$ is increasing in $z$, so $g(1)$ is its minimum.

$$
\begin{aligned}
& g(1)=\frac{(1+u) \cdot a_{n \mid i}-\frac{(1+u)-1}{u}}{a_{n-1 \mid i}}-1=\frac{(1+u) \cdot a_{n \mid i}-1}{a_{n-1 \mid i}}-1= \\
& =\frac{(1+u) \cdot a_{n \mid i}-\left(1+a_{n-1 \mid i}\right)}{a_{n-1 \mid i}}=\frac{(1+u) \cdot a_{n \mid i}-\ddot{a}_{n \mid i}}{a_{n-1 \mid i}}=\frac{(1+u) \cdot a_{n \mid i}-a_{n \mid i} \cdot(1+i)}{a_{n-1 \mid i}}=\frac{a_{n \mid i}}{a_{n-1 \mid i}} \cdot(u-i)
\end{aligned}
$$

So, 


$$
p<\frac{a_{n \mid i}}{a_{n-1 \mid i}} \cdot(u-i)
$$

The condition (c.1) for fixed installments $R$ is looser than the correspondent condition (3) for any installment profile, because

$$
\frac{1}{1+i} \cdot(u-i)<\frac{a_{n \mid i}}{a_{n-1 \mid i}} \cdot(u-i)
$$

since $\frac{1}{1+i}<1$ and $\frac{a_{n \mid i}}{a_{n-1 \mid i}}>1$.

- Let early resolution occur at the date $z$, with $k+1 \leq z<n$ for lessee's insolvency after at least $k$ unpaid payments:

if $0 \leq m \leq i<u: \quad$ Let substitute $A=R \cdot a_{n \mid i}$ in (7.1)

$$
\begin{gathered}
p<R \cdot a_{n \mid i} \cdot \frac{(1+u)^{k+1}-(1+i)^{k+1}}{\sum_{s=k+2}^{n} \frac{R}{(1+i)^{s-(k+1)}}} \\
p<\frac{a_{n \mid i}}{a_{n-(k+1) \mid i}} \cdot\left[(1+u)^{k+1}-(1+i)^{k+1}\right]
\end{gathered}
$$

if $i<m<u: \quad$ Let substitute $A=R \cdot a_{n \mid i}$ in (7.2)

$$
\begin{aligned}
& p<\left[R \cdot a_{n \mid i}-\sum_{s=1}^{n} \frac{R}{(1+m)^{s}}\right] \cdot \frac{\left[(1+u)^{k+1}-(1+i)^{k+1}\right]}{\sum_{s=k+2}^{n} \frac{R}{(1+i)^{s-(k+1)}}} \\
& p<\frac{\left(a_{n \mid i}-a_{n \mid m}\right)}{a_{n-(k+1) i}} \cdot\left[(1+u)^{k+1}-(1+i)^{k+1}\right]
\end{aligned}
$$

- Let early resolution occur at the date $z$, with $k+1 \leq z<n$, for lessee's insolvency after a period of $r$ regularly paid installments, with $r$ known at epoch 0 , and a subsequent period of at least $\mathrm{k}$ unpaid payments. The first admissible resolution date for insolvency $z=r+k+1$ :

if $0 \leq m \leq i<u: \quad$ Let substitute $A=R \cdot a_{n \mid i}$ in (11.1)

$$
p<R \cdot a_{n \mid i} \cdot \frac{(1+u)^{r+k+1}-(1+i)^{r+k+1}}{\sum_{s=k+2}^{n} \frac{R}{(1+i)^{s-(r+k+1)}}}=\frac{a_{n \mid i}}{a_{n-(r+k+1) \mid i}} \cdot\left[(1+u)^{r+k+1}-(1+i)^{r+k+1}\right]
$$

if $i<m<u: \quad$ Let substitute $A=R \cdot a_{n \mid i}$ in (11.2) 


$$
\begin{gathered}
p<\left[R \cdot a_{n \mid i}-\sum_{s=1}^{n} \frac{R}{(1+m)^{s}}\right] \cdot \frac{(1+u)^{r+k+1}-(1+i)^{r+k+1}}{\sum_{s=r+k+2}^{n} \frac{R}{(1+i)^{s-(r+k+1)}}} \\
\quad p<\frac{\left(a_{n \mid i}-a_{n \mid m}\right)}{a_{n-(r+k+1) \mid i}} \cdot\left[(1+u)^{r+k+1}-(1+i)^{r+k+1}\right] .
\end{gathered}
$$

\section{Copyrights}

Copyright for this article is retained by the author(s), with first publication rights granted to the journal.

This is an open-access article distributed under the terms and conditions of the Creative Commons Attribution license (http://creativecommons.org/licenses/by/4.0/). 